\title{
Trastorno de juego por Internet y función parental en estudiantes universitarios
}

\author{
Sebastián Guillermo Risco Martínez(D', Mónica De Los Milagros Cassaretto Bardales(D) \\ I Pontificia Universidad Católica del Perú
}

\section{RESUMEN}

Introducción: las adicciones comportamentales, y en particular la adicción a los videojuegos, están mostrando un incremento notable en los últimos años, por lo que ya es considerada como un trastorno por la Organización Mundial de la Salud (OMS). Este trastorno se ha estudiado en relación con diversas variables, aunque resalta la importancia del contexto familiar, por lo cual se recomienda profundizar en aspectos específicos sobre la relación entre padres e hijos puede y el rol que ello puede tener en el desarrollo de dichas adicciones. Objetivo: analizar la relación entre el riesgo a desarrollar el Trastorno de Juego por Internet (IGD) y la percepción que un grupo de jóvenes universitarios tiene sobre la función parental (materna y paterna). Método: participaron 94 estudiantes universitarios de facultades del área de Ciencias, de Lima, Perú. Se utilizó la prueba de Trastorno de Juego por Internet - IGD-20 (Pontes et al., 2014) y la prueba de Función Parental Adolescente - AFP (Vazsonyi et al., 2003). Resultados: se encontraron asociaciones significativas entre ambos constructos en relación con la función materna, más no con la función paterna. Aquí aparece una correlación positiva entre el IGD y la percepción de soporte de la madre $(r=.24, p<.05)$ y negativa con la comunicación $(r=-.31, p<.01)$ y el monitoreo $(r=-.20, p<.05))$. Discusión y conclusiones: las asociaciones halladas sugieren la importancia del rol de la madre en el desarrollo de las conductas adictivas asociadas a los videojuegos, también se hipotetiza explicaciones por las cuales la función paterna no parece relacionarse con el riesgo IGD.

Palabras clave: juego por Internet, función parental, IGD-20.

\begin{abstract}
Introduction: behavioral addictions, and particularly video game addiction, are showing a notable rise in recent years, which is why the latter is already considered a disorder by the WHO. This disorder has been researched in relation to various variables highlighting the importance of the family context; which is why it's recommended to delve deeper regarding specific aspects about the role that the relationship between parent and child can have in the development of said addictions. Objective: Analyze the relation between the risk of developing Internet gaming disorder (IGD) and the perception a group of university students have of their parent's parenting process (maternal and paternal). Method: 94 university students belonging to science faculties from Lima, Perú participated. The Internet Gaming Disorder Test - IGD-20 (Pontes et al., 2014) and the Adolescent Family Process scale - AFP (Vazsonyi et al., 2003) were used. Results: significant associations between both constructs were found in relation to the mother's parenting, but not with the father's parenting. It shows a positive correlation between IGD and the perception of support from the mother $(r=.24, p<.05)$ and negative ones with communication $(r=-.31, p<.01)$ and monitoring $(r=-.20, p<.05)$. Discussion and conclusions: The associations found suggest the importance of the maternal role in the development of addictive behaviors associated to videogames, also potential explanations as for why the father's parenting doesn't seem to be related with the risk of IGD is hypothesized.
\end{abstract}

Keywords: Internet Gaming Disorder, Parenting, IGD-20.

\footnotetext{
Autor de correspondencia:

Sebastián Guillermo Risco Martínez.

Correo electrónico: a20110421@pucp.pe

Recibido: 24 de febrero de 2021

Aceptado: 21 de julio de 2021

doi: 10.28931/riiad.2021.2.04
} 


\section{INTRODUCCIÓN}

La OMS reconoció la adicción a los videojuegos como un trastorno en el 2019, clasificación que tendrá efecto a partir del 2022 (Hoggins, 2019). Existe evidencia de un importante incremento de este tipo de adicciones (Centro de Información y Educación para la Prevención del Abuso de Drogas [CEDRO], 2018; Hull, 2020), pues cada año se reportan más casos de adolescentes y adultos jóvenes que han abandonado sus estudios, sus trabajos, e inclusive sus familias para dedicarse exclusivamente al juego (Salvarli \& Griffiths, 2019). La adicción a los videojuegos, también conocida como Trastorno de Juego por Internet (IGD), puede llevar a graves consecuencias en la vida de las personas. En poblaciones jóvenes, en particular, puede llevar al fracaso académico y la pérdida de interés en otras actividades o aficiones, así como al deterioro de las actividades sociales y familiares (APA, 2013).

Pontes y colaboradores (2014) retoman el modelo de adicciones de Griffiths (2005) y lo aplican a las adicciones a los videojuegos. Estos autores consideran que dicha adicción involucra seis dimensiones: prominencia, la importancia que el videojuego tiene para la persona; abstinencia, malestar que surge cuando la persona no juega videojuegos; tolerancia, necesidad de incrementar la cantidad de juego para sentir placer; recaída, intentos fallidos de intentar controlar la conducta; modificación del humor, uso de los videojuegos para escapar de emociones negativas; y conflicto, que es la interferencia con otras áreas de la vida, como la social, laboral, académica, y la salud.

Los reportes de prevalencia del IGD varían acorde al contexto, pues se calcula que el porcentaje de personas con IGD se encuentra entre $0.7 \%$ y $27.5 \%$ en diferentes países a nivel mundial (Przybylski et al., 2016; Salvarli \& Griffiths, 2019); posiblemente esto se deba al uso incrementado de la tecnología, lo que ocasiona que el consumo del videojuego se vuelva más accesible a las personas (Lopez-Portillo, 2018). Además, el aislamiento o distanciamiento social propio del contexto mundial, actual producto de la pandemia, puede estar acentuando esta tendencia, particularmente en jóvenes (Fernandes et al., 2020; Hull, 2020).

Si bien, el consumo de videojuegos se da en cualquier etapa de la vida, suele iniciarse tempranamente; de manera que el grupo de adolescentes y jóvenes adultos son los que presentan las tasas más elevadas de IGD (Limelight Networks, 2018). Por ello, los universitarios son un potencial grupo de riesgo, ya que en esta etapa transitan por edades en las que suelen contar con mayor autonomía y menor control para el uso de Internet y videojuegos por parte de los padres (Vieira et al., 2020).
Se han realizado numerosos estudios sobre el IGD y su vínculo con ciertas características sociodemográficas o patrones de juego. Respecto al sexo, se ha encontrado mayor prevalencia en los hombres que en las mujeres (Bender \& Gentile, 2019; Carlisle, 2017; Wichstrom et al., 2019). Asimismo, Beard y colaboradores (2017) encontraron que el riesgo a desarrollar IGD es mayor cuanto más temprano es el inicio en esta actividad y cuantas más horas se les dedican.

Por otro lado, se ha encontrado que los problemas de IGD de los adolescentes pueden estar asociados al ambiente familiar y, en particular, a la relación progenitor-adolescente (Bonnaire et al., 2019). En esa línea, existen estudios que indican que los padres tienen un rol importante en la prevención de desórdenes relacionados con los videojuegos, pues son estos los que ponen las pautas para el uso de videojuegos e Internet (Su et al., 2018). Además, existe evidencia de que dicho monitoreo predice el desarrollo de síntomas de IGD y de adicción a Internet (Koning et al., 2018; Vieira et al., 2020).

Por ello, el papel de los padres en la educación de sus hijos se torna significativo como un factor de riesgo o de protección ante el desarrollo de cualquier adicción (Wichstrom et al., 2019). Vazsonyi, Hibbert y Snider (2003) proponen un concepto ligado a ese rol que tienen los padres y lo llaman función parental. Este se define como la actuación que tienen los padres en la crianza y en la satisfacción de las necesidades de sus hijos, a partir del tipo de relación que mantienen con ellos. Esta puede evaluarse con base en seis dimensiones o aspectos importantes de la relación que tiene cada padre con su hijo o hija: Comunicación, que se refiere a todo intercambio de información entre los padres y los hijos; Cercanía, que es la sensación de proximidad emocional y comprensión entre padres e hijos; Soporte, que abarca la oferta de apoyo, aceptación y escucha a los hijos; Monitoreo, que involucra el conocimiento y supervisión de los padres sobre las actividades que los hijos realizan; Aprobación de los pares, definido como la percepción que los padres tienen sobre los pares o amistades que sus hijos frecuentan; y Conflicto, que es el grado de tensión que existe entre padres e hijos. La función parental se ha estudiado con relación al consumo de drogas (Oficina de Naciones Unidas contra la Droga y el Delito [UNODC], 2015), donde se encontró que los consumidores obtuvieron puntajes estadísticamente menores en casi todas las dimensiones; sin embargo, son pocos los estudios que vinculan la dinámica del ámbito familiar y el IGD.

El rol de los papás consiste en ayudar a sus hijos a regularse y evitar que tengan conductas que puedan perjudicar su bienestar; en ese sentido, su participación en la crianza es clave para comprender la génesis de la adicción, así como para dar insumos claves para la inter- 
vención con la familia. Si bien el papel de los padres se considera importante, aún queda por dilucidar qué aspectos específicos de dicha relación son las vinculadas al IGD, por lo que es necesario identificar las diferencias de la misma con respecto a cada progenitor.

En este contexto, el objetivo de la investigación fue analizar la relación entre el riesgo a desarrollar Trastorno de Juego por Internet y la percepción de la función parental del padre y la madre, en un grupo de estudiantes universitarios limeños de las carreras de Ciencias. Adicionalmente, se buscó analizar la relación entre el riesgo de desarrollar el trastorno y las variables sociodemográficas, como también los hábitos de juego

\section{MÉTODO}

\section{Participantes}

El grupo de participantes estuvo conformado por 94 estudiantes del área de ciencias de una universidad privada de Lima, Perú, usuarios de videojuegos por Internet, de los cuales $73 \%$ eran varones y $27 \%$ mujeres, con edades entre los 18 y 23 años ( $X=18.99, D E=1.15)$. Como criterio de inclusión, se tomó en consideración que hubieran usado videojuegos al menos una vez en el último año. 13\% reportó que vivía solo, mientras que $87 \%$ reportó vivir con familiares. Con respecto al juego, $68 \%$ reportó jugar videojuegos en la actualidad y $26 \%$ que tendía a jugar solo, a diferencia del $74 \%$ que jugaba con amigos de manera presencial o virtual. La edad de inicio reportada osciló entre los 2 y 17 años ( $M=9.7, D E=3.4)$ y el tiempo promedio de juego fue de 6 horas a la semana.

Se tomaron en cuenta las consideraciones éticas a través de un consentimiento informado, donde se explicaron los objetivos de la investigación y se remarcó el carácter anónimo de su participación. Los instrumentos fueron aplicados de manera virtual en un aula de clases, al compartir el link para acceder al formulario de Google creado para esta investigación.

\section{Instrumentos}

La medición del riesgo de adicción a los videojuegos se realizó con la prueba del Trastorno de Juego por Internet 20 (Internet Gaming Disorder 20, IGD-20) desarrollada por Pontes y colaboradores (2014). La escala cuenta con 20 ítems, en un formato de respuesta tipo Likert de 5 puntos, incluye seis dimensiones: Prominencia, Abstinencia, Tolerancia, Recaída, Modificación del humor y Conflicto. El mayor puntaje en la prueba supone un mayor riesgo de desarrollar el trastorno. Los autores validaron la escala con 1,003 jugadores de videojuegos de 57 países.
La evaluación de la Función Parental se realizó a través del AFP (Adolescent Family Process), creada por Vazsonyi y colaboradores (2003). Cuenta con 50 ítems, de los cuales 25 se abocan a la relación con el padre y los otros 25 a la relación con la madre. Los ítems son de tipo Likert de 5 puntos organizados en seis dimensiones: Cercanía, Soporte, Monitoreo, Comunicación, Conflicto y Aprobación de pares para cada uno de los padres. La escala fue validada por los autores con 5,810 adolescentes y jóvenes de Estados Unidos y Europa. La AFP fue adaptada para la población peruana por Molinero (2006), con una muestra de 276 adolescentes de secundaria de cuatro instituciones educativas de Lima.

\section{Análisis de datos}

Para los análisis estadísticos de la información recogida, se utilizó el programa SPSS 23.0. En primer lugar, se examinó la confiabilidad de los instrumentos mediante el coeficiente de alfa. Se analizó la normalidad de los datos, y en función de ellos se precedió a usar las pruebas paramétricas. Las correlaciones entre los instrumentos se realizaron con el coeficiente $r$ de Pearson y las diferencias entre subgrupos, con la t de Student y ANOVA.

\section{RESULTADOS}

En primer lugar, se analizó la relación entre el IGD y la función parental (Tabla 1), donde sólo se encontraron asociaciones significativas con la función de la madre, entre las que destacan, con riesgo de desarrollar IGD, las asociaciones entre las funciones de Comunicación, Soporte y Monitoreo, la primera directa y las dos últimas inversas. De esta manera, a mayor Soporte y menor Comunicación y Monitoreo, mayor será el riesgo de desarrollar IGD. Por otro lado, se encuentra que las subáreas del IGD con mayores vínculos con función materna son de Abstinencia y Recaída del IGD. Otras asociaciones inversas encontradas fueron entre la Comunicación y la prominencia y Conflicto, mientras que otras directas fueron entre el Monitoreo y la Abstinencia, y el Conflicto con la Recaída.

Con el fin de explorar si existen otras variables vinculadas al riesgo de desarrollar IGD, se analizó su relación con las variables sociodemográficas, en cuyo caso sólo se encontraron diferencias en función al sexo de los participantes y a la condición de vivir solos. En el primer caso, los varones presentaron un mayor nivel de riesgo, especialmente en las áreas de Prominencia y Recaída. Asimismo, quienes viven solos presentan un mayor riesgo de desarrollar IGD, en particular en la dimensión de Tolerancia (Tabla 2). No se hallaron relaciones con otras 
variables reportadas en la ficha de datos como el género de videojuegos, autopercepción de rendimiento, si tiene pareja, si juega solo o con amigos, percepción de salud y si ha tenido tratamiento psicológico.

Con relación a las variables asociadas a los hábitos de juego, se hallaron varias diferencias. Quienes reportaron que jugaban videojuegos actualmente presentaron mayor riesgo de IGD; específicamente mostraron mayor Prominencia, Ánimo, Tolerancia y Recaída que quienes no reportaron jugar actualmente (Tabla 3).
Con respecto a la edad de inicio en los videojuegos, se hallaron varias relaciones inversas con el IGD, donde a menor edad de inicio, mayor riesgo de IGD ( $r=-.31$, $p<.01)$, en particular en los factores Prominencia $(r=-.25$, $p<.05)$ y Conflicto $(r=-.23, p<.05)$.

Finalmente, el tiempo dedicado al juego también muestra asociaciones con el riesgo a desarrollar el trastorno. Se encontró que, a mayor número de horas de juego, particularmente durante días de clase, existe mayor riesgo de IGD y sus dimensiones (Tabla 4).

Tabla 1

Correlaciones entre el IGD y la función materna

\begin{tabular}{|c|c|c|c|c|c|}
\hline Función materna & IGD Total & Prominencia & Abstinencia & Conflicto & Recaída \\
\hline Soporte & $.24^{*}$ & $.27^{\star \star}$ & $.29^{\star \star}$ & & $.27^{\star \star}$ \\
\hline Monitoreo & $-.20^{\star}$ & & $-.24^{*}$ & & $-.21^{\star}$ \\
\hline Comunicación & $-.31^{* \star}$ & $-.24^{*}$ & $-.30^{* *}$ & $-.31^{\star \star}$ & $-.26^{*}$ \\
\hline Conflicto & & & & & $.26^{*}$ \\
\hline
\end{tabular}

${ }^{*} p<.05 ;{ }^{* *} p<.01$ Nota: no se encontraron correlaciones entre el IGD y la función paterna

Tabla 2

Diferencias en el IGD de acuerdo con las variables sociodemográficas

\begin{tabular}{|c|c|c|c|c|c|}
\hline & & Media & D.E. & $t$ & $d$ \\
\hline \multirow{2}{*}{ Riesgo de IGD } & Varones & 40.17 & 12.13 & \multirow{2}{*}{$3.12^{\star \star}$} & \multirow{2}{*}{.66} \\
\hline & Mujeres & 33.16 & 8.54 & & \\
\hline \multirow{2}{*}{ Prominencia } & Varones & 6.23 & 2.47 & \multirow{2}{*}{$4.08^{* \star *}$} & \multirow{2}{*}{.86} \\
\hline & Mujeres & 4.4 & 1.68 & & \\
\hline \multirow{2}{*}{ Recaída } & Varones & 5.72 & 2.4 & \multirow{2}{*}{$3.98^{\star \star \star}$} & \multirow{2}{*}{.79} \\
\hline & Mujeres & 4.2 & 1.26 & & \\
\hline \multirow{2}{*}{ Riesgo de IGD } & Vive solo/a & 46.91 & 14.41 & \multirow{2}{*}{$2.89^{\star \star}$} & \multirow{2}{*}{.79} \\
\hline & Con Familiares & 36.82 & 10.76 & & \\
\hline \multirow{2}{*}{ Tolerancia } & Vive solo/a & 7.00 & 2.34 & \multirow{2}{*}{$3.30^{* * *}$} & \multirow{2}{*}{.97} \\
\hline & Con Familiares & 4.88 & 1.97 & & \\
\hline
\end{tabular}

Varones: $\mathrm{N}=69$, Mujeres: $\mathrm{N}=25$

Viven solos: $\mathrm{N}=12$, Viven con familiares: $\mathrm{N}=80$

${ }^{*} p<.05 ;{ }^{* *} p<.01 ;{ }^{* *} p<.001$ 
Tabla 3

Diferencias en el IGD entre quienes utilizan videojuegos actualmente y quienes no

\begin{tabular}{|c|c|c|c|c|c|}
\hline & & Media & D.E. & $t$ & $d$ \\
\hline Riesgo de IGD & $\begin{array}{l}\text { No } \\
\text { Sí }\end{array}$ & $\begin{array}{l}33.30 \\
40.66\end{array}$ & $\begin{array}{l}10.82 \\
11.37\end{array}$ & $-2.97^{\star *}$ & .66 \\
\hline Prominencia & $\begin{array}{l}\text { No } \\
\text { Sí }\end{array}$ & $\begin{array}{l}4.70 \\
6.23\end{array}$ & $\begin{array}{l}2.10 \\
2.42\end{array}$ & $-2.99^{\star *}$ & .67 \\
\hline Ánimo & $\begin{array}{l}\text { No } \\
\text { Sí }\end{array}$ & $\begin{array}{l}6.57 \\
8.16\end{array}$ & $\begin{array}{l}2.57 \\
2.90\end{array}$ & $-2.56^{*}$ & .58 \\
\hline Tolerancia & $\begin{array}{l}\text { No } \\
\text { Sí }\end{array}$ & $\begin{array}{l}4.50 \\
5.55\end{array}$ & $\begin{array}{l}1.91 \\
2.17\end{array}$ & $-2.26^{*}$ & .51 \\
\hline Recaída & $\begin{array}{l}\text { No } \\
\text { Sí }\end{array}$ & $\begin{array}{l}4.33 \\
5.78\end{array}$ & $\begin{array}{l}1.56 \\
2.39\end{array}$ & $-3.51^{\star \star \star}$ & .71 \\
\hline
\end{tabular}

Juegan actualmente: $\mathrm{N}=64$, No juegan actualmente: $\mathrm{N}=30$

${ }^{\star} p<.05 ;{ }^{* \star} p<.01 ;{ }^{* \star *} p<.001$

Tabla 4

Correlaciones entre el IGD y las horas de juego

\begin{tabular}{|c|c|c|c|}
\hline & Durante días de clase & Durante fines de semana & Durante vacaciones \\
\hline Riesgo de IGD & $.41^{* *}$ & $.31^{\star *}$ & $.45^{\star *}$ \\
\hline Prominencia & $.44^{\star \star}$ & $.35^{\star \star}$ & $.52^{\star \star}$ \\
\hline Tolerancia & $.35^{\star \star}$ & & $.32^{\star \star}$ \\
\hline Abstinencia & $.29^{\star \star}$ & $.21^{*}$ & $.25^{*}$ \\
\hline Conflicto & $.30^{\star *}$ & $.32^{\star *}$ & $.35^{\star *}$ \\
\hline Recaída & $.47^{\star *}$ & $.33^{* *}$ & $.48^{* *}$ \\
\hline
\end{tabular}

${ }^{*} p<.05 ;{ }^{* *} p<.01 ;{ }^{* *} p<.001$

\section{DISCUSIÓN Y CONCLUSIONES}

El número de personas dependientes de los videojuegos se encuentra en crecimiento (CEDRO, 2018). Dado que este es un pasatiempo legal, de fácil acceso y puede ser de bajo costo, cuesta tomar medidas para forzar a la persona a limitar su consumo. Bajo esa línea, existen recomendaciones de que el abordaje terapéutico incluya un enfoque hacia la familia y el tipo de interacciones que establecen (Bonnaire et al., 2019).

De acuerdo con lo hallado en la presente investigación, la relación con la madre y la forma en que el hijo percibe su función se puede asociar con el riesgo de IGD, más no existirían relaciones en la función paterna y el IGD. La ausencia de asociaciones con la función del padre podría sugerir una mayor presencia de la madre en la crianza y cuidado de los hijos. Existe evidencia que muestra que en las culturas latinas son las madres quienes suelen dedicar mayor tiempo a la tarea de crianza que los padres (Mejía \& López, 2010; Zurita et al., 2017).

Con respecto a las relaciones entre IGD y función materna, resaltan aquellas con las áreas Comunicación, Soporte y Monitoreo. Ello iría en la línea de lo encontrado por Koning y colaboradores (2018), quienes plantean que las respuestas parentales efectivas en el manejo del Internet predicen la presencia de síntomas vinculados al IGD. Dentro de las prácticas recomendadas se encuentran: la frecuencia y calidad de la comunicación sobre el Internet entre padres e hijos; el establecimiento 
y monitoreo de las reglas sobre el uso del Internet, y las restricciones en su uso.

De manera específica, se encuentra que la Comunicación con la madre se relaciona de manera negativa con IGD, lo que coincide con lo reportado en otros estudios (Adams et al., 2018; Seong, 2017). Uno de los principales factores que puede llevar al abuso de videojuegos, es el deseo de escapar de la realidad debido a que esta es percibida como muy estresante, y si el joven no cuenta con otra persona a la cual pueda recurrir para liberar sus preocupaciones o escuchar sus problemas, éste posiblemente acudirá a los videojuegos con el fin de escapar de sus problemas y cubrir su necesidad de comunicación con otros en línea (Gonzalvez et al., 2016; Mascaraque, 2015).

De este modo, la escasa comunicación se asocia con un incremento de las dimensiones de Conflicto del IGD, lo que podría favorecer el IGD en áreas específicas como la Abstinencia y Recaída. Esto se podría entender desde lo que plantean Deleuze y colaboradores (2019), que indican que la escasa comunicación con los hijos hace que los padres no logren detectar cuando el consumo de juego está llegando al abuso.

Sobre la correlación positiva entre el IGD y el Monitoreo de la madre, puede estar asociada con el hecho de que es ella quien suele estar más involucrada en la regulación del consumo habitual de los videojuegos del hijo. La mayoría de las investigaciones señalan la importancia del monitoreo del comportamiento, especialmente en las actividades ligadas al uso del Internet y videojuegos, debido a que esto puede tener un efecto en la reducción del riesgo de desarrollar IGD (Koning et al., 2018; Vieria et al., 2020).

La dimensión de Soporte de la madre, definida como la oferta de apoyo, aceptación y escucha a los hijos, tuvo una relación directa con el IGD. Este resultado podría explicarse desde la percepción del hijo, de que la madre le seguirá brindando apoyo incluso cuando el consumo de videojuegos ya esté interfiriendo con otras áreas de su vida (UNODC, 2015). Clancy (2017) plantea que los padres pueden actuar como "habilitadores"; es decir, ofrecer ayuda en cosas que podría y debería asumir el hijo y tienden a resolverles los problemas que se presentan en su camino (Khaleghi, 2012), lo que podría llevar a que éste sienta que existe alguien que solucionará las dificultades y no permitirá que vea el incentivo de limitar su consumo y siga desarrollando su dependencia (Clancy, 2017; Khaleghi, 2012).

Con relación a las variables sociodemográficas, las diferencias en función al sexo mostraron que los varones presentan mayor riesgo a desarrollar el Trastorno de Juego por Internet que las mujeres, lo cual concuerda con lo reportado en otros estudios sobre el IGD (Bender
\& Gentile, 2019; Carlisle, 2017; Wichstrom et al., 2019). El vivir solos va en el mismo sentido, lo que confirma el papel de la familia como elemento protector frente a una posible adicción y ello se refleja en lo encontrado por Vieira (2020), quien reportó que los estudiantes que aseguraron tener cualquier tipo de control parental sobre su uso de Internet presentaban menor probabilidad de desarrollar adicciones relacionadas con el Internet.

Tal como se esperaba, se halló relación entre las características del uso del videojuego y el IGD. De este modo, se encontró que quienes utilizan videojuegos actualmente (durante el semestre académico) presentan mayores puntajes en el IGD y la mayor parte de sus componentes que quienes no lo hacen. Lo mismo sucede con el tiempo invertido en jugar, donde a mayor tiempo mayor riesgo de desarrollar el trastorno. Finalmente, cuanto más temprano es el inicio en los videojuegos, mayor es el riesgo de una adicción. Wichstrom y colaboradores (2019), sugieren que este inicio temprano puede llevar a que los niños se acostumbren a su uso como una forma de entretenimiento, que conducirá a un incremento en su uso y con ello a una dependencia temprana.

Es importante resumir las principales limitaciones del presente estudio, entre ellas el contar con un número limitado de participantes, por lo que es posible que esto no permitiera visibilizar algunas relaciones potenciales entre las variables y contar con una muestra que presenta varias condiciones de protección para el desarrollo del IGD, como el ser estudiante de una universidad privada. Los próximos estudios podrían involucrar a jóvenes en contextos distintos al académico, por ejemplo, en cabinas de Internet o a través de redes sociales.

Los resultados de este estudio brindan algunos insumos para tomar en cuenta en las iniciativas que busquen fomentar la prevención de las adicciones a los videojuegos en jóvenes. Bajo esta lógica, se rescata el rol de la familia, particularmente el de la madre, como agente protector, con lo cual se puede fortalecer acciones dirigidas hacia ellas.

De esta manera, es importante reforzar algunas de las pautas de crianza materna, en especial aquellas orientadas a la regulación del comportamiento asociado a los videojuegos desde que empiezan a ser usados por el niño en etapas tempranas (Bonnaire et al., 2019). Dicha supervisión debería disminuir a medida que se ingrese a la adolescencia, por lo que resultarán menos efectivas las aproximaciones restrictivas durante estos periodos (Koning et al., 2018). Ello implica que los padres necesitan ser conscientes que los videojuegos son herramientas usadas tanto en el entretenimiento como en la formación del menor, por lo que su acceso y uso deben ser claramente regulados por el adulto cuidador; en ese sentido es importante que los padres conozcan 
el tipo de videojuego que el hijo desea jugar antes de autorizarlo, además, deben establecer reglas para su uso basado en aspectos ligados a la edad, horarios o contenido; asimismo, su restricción debe estar asociada a aspectos como el desempeño académico y social del hijo. Una tarea importante es buscar espacios para acompañarlos durante el uso inicial del videojuego, así como monitorear su comportamiento durante la etapa escolar.

Por otro lado, es importante que las madres establezcan relaciones de apoyo con los hijos y que diferencien entre comportamientos que brindan soporte de los que favorecen la adicción, ya que se pueden promover involuntariamente actitudes de codependencia cuando la madre evita que su hijo asuma las consecuencias de su comportamiento de abuso del videojuego (Khaleghi, 2012). También, es importante fomentar habilidades de comunicación a nivel familiar, especialmente en la etapa de la adolescencia, donde es necesario disponer de espacios de apertura y escucha, y que se promueva un estilo parental democrático, con base en una relación cálida y colaborativa. Es necesario desarrollar un estilo de comunicación efectiva desde la niñez para que en la adolescencia sea menor la predisposición al uso excesivo del Internet como único medio de distracción y juego (Bonnaire et al., 2019).

Por último, una dinámica familiar equilibrada implica que las responsabilidades y tareas del hogar sean compartidas por ambos padres, aunque el padre no pareciera ser significativo en dicho estudio, éste contribuye como fuente de soporte tanto para el hijo como para la madre, de esta manera es necesario un involucramiento parental en las tareas de crianza

\section{REFERENCIAS}

Adams, B., Stavropoulos. V., Burleigh. T., Liew. L., Beard. C., \& Griffiths. M. (2018). Gaming Disorder Behaviors in Emergent Adulthood: a Pilot Study Examining the Interplay Between Anxiety and Family Cohesion. International Journal of Mental Health and Addiction. 1-17. doi: 10.1007/s11469-018-9873-0

American Psychiatric Association. (2013). Diagnostic and Statistical Manual of Mental Disorders (5th ed.). Arlington. VA: Author.

Beard, C., Haas, A., Wickman, R., \& Stavropoulos, V. (2017). Age of initiation and Internet gaming disorder: the role of self-esteem. Cyberpsychology. behavior and social networking 20(6). 397401. doi: 10.1089/cyber.2017.0011.

Bender, P., \& Gentile, D. (2019). Internet gaming disorder: Relations btween needs satisfaction in-game and in life in general. Psychology of Popular Media Culture. Advance online publication.

Bonnaire, C., Liddle, H., Har, A., Nielsen, P., \& Phan, O. (2019). Why and how to include parents in the treatment of adolescents presenting Internet gaming disorder? Journal of Behavioral Addictions 8(2). 201-212. doi: 10.1556/2006.8.2019.27
Carlisle, K. (2017). Personality, motivation and Internet gaming disorder: understanding the addiction. Doctor of Philosophy (PhD). Dissertation, Counseling and Human Services. Old Dominion University. doi: 10.25777/n9br-q768

Centro de Información y Educación para la Prevención del Abuso de Drogas (2018). El problema de drogas en el Perú 2018. Recuperado de: http://www.repositorio.cedro.org.pe/bitstream/CEDRO/378/1/CEDRO.Problema\%20de\%20las\%20drogas.2018.pdf

Clancy, C. (2017). How to stop enabling an addict. Recuperado de: https://journeypurebowlinggreen.com/stop-enabling/

Deleuze, J., Maurage, P., Schimmenti, A., Nuyens, F., Melzer, A., \& Billieux, J. (2019). Escaping reality through videogames is linked to an implicit preference for virtual over real-life stimuli. Journal of Affective Disorders 245. 1024-1031.

Fernandes, B., Nanda, Urmi., Tan-Mansukhani, R., Velljo, A., \& Essau, C. (2020). The impacto of COVID-19 lockdown on Internet use and escapism in adolescents. Revista de Psicología Clínica con Niños y Adolescentes 7(3). 59-65. doi: 10.21134/rpcna.2020.mon.2056

Griffiths, M. (2005). A 'components' model of addiction within a biopsychosocial framework. Journal of Substance Use 10(4). 191197. doi: 10.1080/14659890500114359

Gonzalvez, M., Espada, J., Guillen-Riquelme, A., Secades, R., \& Orgiles, M. (2016). Asociación entre rasgos de personalidad y consumo de sustancias en adolescentes españoles. Addicion 28(2). 108-115.

Hoggins, T. (2019). World Health Organization adopts 'gaming addiction' as official illness despite industry opposition. Recuperado de: https://www.telegraph.co.uk/gaming/news/world-health-organisation-adopts-gaming-addiction-official-illness/

Hull, M. (2020). Video Game Addiction Statistics. Recuperado de: https://www.therecoveryvillage.com/process-addiction/video-game-addiction/related/gaming-addiction-statistics/

Khaleghi, K. (2012). Are you empowering or enabling? Recuperado de: https://www.psychologytoday.com/us/blog/the-anatomy-addiction/201207/are-you-empowering-or-enabling

Koning, I., Peeters, M., Finkenauer, C., \& Van Den Eijnden, R. (2018). Bidirectional effects of Internet-specific parenting practices and compulsive social media and Internet game use. Journal of Behavioral Addictions 7(3). 624-632. doi: 10.1556/2006.7.2018.68

Limelight Networks, (2018). The State of Onine Gaming 2018. Recuperado de: https://www.limelight.com/resources/white-paper/ state-of-online-gaming-2018/

Lopez-Portillo, J. (2018). La gran transición. Retos y Oportunidades del cambio tecnológico exponencial. México: Fondo de Cultura Económica.

Mascaraque, A. (2015). Inteligencia emocional y consumo de sustancias en una muestra de mujeres universitarias ¿cómo se relacionan? España: Autor.

Mejía, L., \& López, L. (2010). La familia y la cultura: una conexión innovadora para el cuidado de la salud. Recuperado de: http://scielo.isciii. es/scielo.php?script=sci_arttext\&pid=\$1132-12962010000200015 
Molinero, C. (2006). Adaptación de la escala "Adolescent Family Process" (AFP) en una muestra de adolescentes de Lima Metropolitana y Callao. (Tesis de pregrado). Pontificia Universidad Católica del Perú, Lima.

Pontes, H., Király, O., Demetrovics, Z., \& Griffiths, MD. (2014). The conceptualization and measurement of DSM-5 Internet gaming disorder: the development of the IGD-20 test. PLOS ONE 9(10). doi: 10.1371/ journal.pone.0110137

Przybylski, A., Weinstein, N., \& Murayama. K. (2016). Internet Gaming Disorder: Investigating the Clinical Relevance of a New Phenomenon. The American journal of psychiatry 174(3). 230-236. doi: 10.1176/appi.ajp.2016.16020224

Salvarli, Ş., \& Griffiths, M. (2019). Internet gaming disorder and its associated personality traits: A systematic review using prisma guidelines. International Journal of Mental Health and Addiction 2019. 1-23.

Seong, Ok. (2017). Developmental process of Internet Gaming Disorder among south korean adolescents: effects of family environment and recreation experience. Journal of Child and Family Studies 26(6). 1527-1535. doi: 10.1007/s10826-017-0686-8

Su, B., Yu, C., Zhang, W., Su, Q., Zhu, J., \& Jiang, Y. (2018). Father-Child Longitudinal Relationship: Parental Monitoring and
Internet Gaming Disorder in Chinese Adolescents. Frontiers in Psychology 9(95). doi: 10.3389/fpsyg.2018.00095

Oficina de Naciones Unidas contra la Droga y el Delito (2015). Función parental paterna y maternal en adolescentes consumidores de drogas, adolescentes infractores de la ley y un grupo control. Perú: Autor.

Vazsonyi, A., Hibbert, J., \& Snider. J. (2003). Exotic enterprise no more? Adolescent reports of family and parenting processes from youth in four countries. Journal of Research on Adolescence 13(2). 129-160. doi: 10.1111/1532-7795.1302001

Vieira, M., Formiga, A., Santos, C., Sousa, D., Resende, C., Campos, R., Nogueira, N., Carvalho, P., \& Ferreira, S. (2020). Adolescent Internet addiction - role of parental control and adolescent behaviours. International Journal of Pediatrics and Adolescent Medicine 7. 116-120

Wichstrom. L., Stenseng. F., Belsky. J., von Soest. T., \& Hygen. B. (2019). Symptoms of Internet Gaming Disorder in Youth: Predictors and Comorbidity. Journal of abnormal child psychology 47(1). 71-83. doi: 10.1007/s10802-018-0422-X.

Zurita, P., Murillo, F., \& Defaz, Y. (2017). Los saberes culturales en la crianza de los hijos. Boletín Virtual 6(6) 